Cinémas

Revue d'études cinématographiques

Journal of Film Studies

\title{
Montage, simultanéité et continuité dans Le Sursis de Sartre
}

\section{Damien François}

Volume 8, numéro 3, printemps 1998

Cinélekta 2

URI : https://id.erudit.org/iderudit/024759ar

DOI : https://doi.org/10.7202/024759ar

Aller au sommaire du numéro

Éditeur(s)

Cinémas

ISSN

1181-6945 (imprimé)

1705-6500 (numérique)

Découvrir la revue

Citer cet article

François, D. (1998). Montage, simultanéité et continuité dans Le Sursis de Sartre. Cinémas, 8(3), 75-103. https://doi.org/10.7202/024759ar
Résumé de l'article

Le Sursis (1945) est un roman de la simultanéité qui, par son agencement discursif, annonce l'esthétique du montage visuel. Sartre y a recours à une écriture cinématographique reposant sur le montage et la continuité qui présupposent une lecture cinématographique. La simultanéité n'existe pas en tant que telle et ne peut naître que dans l'esprit du récepteur qui, de la sorte, participe à la production de sens. 


\section{Montage, simultanéité et continuité dans Le Sursis de Sartre}

\section{Damien François}

\section{RÉSUMÉ}

Le Sursis (1945) est un roman de la simultanéité qui, par son agencement discursif, annonce l'esthétique du montage visuel. Sartre y a recours à une écriture cinématographique reposant sur le montage et la continuité qui présupposent une lecture cinématographique. La simultanéité n'existe pas en tant que telle et ne peut naître que dans l'esprit du récepteur qui, de la sorte, participe à la production de sens.

\section{ABSTRACT}

Le Sursis (1945) is a novel of simultaneity whose discursive structure prefigures the esthetic of visual montage. In it, Sartre's use of a cinematographic writing style relies on montage and continuity, and presupposes a cinematographic reading. Simultaneity does not exist as such, and can only take shape in the mind of the receiver, who thus participates in the production of meaning.

La narration cinématographique classique a imposé un discours sur le monde essentiellement axé sur le montage et la continuité. Grâce au pouvoir de monstration du signifiant filmique - capable de véhiculer concrètement plus de signifié que son homologue littéraire puisqu'il fonctionne en gros selon les mêmes règles que la réalité sensible - il est possible de passer d'un espace-temps à un autre sans que la compréhension à la lecture souffre d'un déficit cognitif. Cette écriture cinématogra- 
phique a eu une influence sur la littérature, dans la mesure où elle lui a permis de renoncer à tout un système de transition et d'explicitation de ces passages, qu'il s'agisse d'une seule série événementielle ou de plusieurs. Mais, dans ce transfert intersémiotique, la "continuité diégétique» du film risque de devenir, en littérature, une "discontinuité diégétique». Une discontinuité qui, à la réception, grâce à la participation active du lecteur dans la création de l'œuvre, peut cependant retrouver une homogénéité.

Sommairement, le montage peut être défini comme la réorganisation et, partant, la transcendance de la perception humaine de l'espace-temps et ce, par sa mise en discours et la libération des contraintes qu'elle impose par sa continuité. De manière générale, on peut donc dire que " [...] le montage, c'est l'irruption de la modernité dans l'art" (Ishaghpour, p. 45). L'art moderne se démarque de la représentation traditionnelle (fondée sur le réalisme, la vraisemblance psychologique, la linéarité, la causalité et la continuité) par l'émergence du paradigme de la discontinuité. La révolution des rapports spatio-temporels représente le paradigme distinctif du roman moderne, le temps comme subjectivité triomphant du temps (utopique) comme objectivité.

Qu'en est-il dans le roman Le Sursis publié par Jean-Paul Sartre en 1945? L'écriture de ce roman est placée sous le signe d'une double inspiration: la narration cinématographique et les grands romanciers américains de la première moitié du $\mathrm{XX}^{c}$ siècle, dont les "romanciers de la simultanéité ", John Dos Passos en tête. Cette deuxième influence a été reconnue par Sartre lui-même, de manière implicite, dans trois textes au moins: "American Novelists in French Eyes", "À propos de John Dos Passos et de 1919" et "Situation de l'écrivain en 1947 ". Il est possible de fournir une explication historique et socioculturelle à ces deux sources d'influence : avec la Seconde Guerre mondiale, la France découvre véritablement "en direct" la culture américaine, le roman et le cinéma y tenant une place de choix ${ }^{1}$. Quoique étiqueté plus tard d' "antiaméricain " ", Sartre a donc lui aussi été séduit par la littérature, le cinéma et le jazz américains. L'autre influence, d'ordre intersémiotique celle-là, est plus diffi- 


\section{VENDREDI 23 SEPTEMBRE}

Seize heures trente à Berlin, quinze heures trante ì Londres. L'hôtel s'ennuyait sur sa colline, désert et solennel, avec un vieillard dedans. A Angoulème, à Marseille, à Gand, a Douvres, ils pen* saient : "Que fait-il? Il est plus de trois heures, pourquoi ne descend-il pas? \#I était assis dans le salon aux persiennes demi-closes, les yeux fixes sous ses épais sourcils, la bouche légèrement ouverte, comme $s^{2}$ il se rappelait un souvenir très ancien. Il ne lisait plus, sa vieille main tavelée, qui tenait encore les feuillets, pendait le long de ses genoux. Il se tourna vers Horace Wilson et demanda: quelle heure est-il? " et Horace Wilson dit : "Quatre heures et demie, i peu près. Le vieillard leva ses gros yeux, eut un petit rire aimable et dit : II fait chaud. Une chaleur rousse, crépitante, pailletée s'était affalée sur l'Europe; les gens avaient de la chaleur sur les mains, au fond des yeux, dans les bronches; ils attendaient, ecceurés de chaleur, de poussière et d'angoisse. Dans le hall de l'hôtel, les journalistes attendaient. Dans la cour, trois chauffeurs attendaient, immobiles au volant de leurs autos; de l'autre cotte tu $\mathrm{Hhin}$, immobiles dans le hall de l'hôtel Dreesen, de longs Prussiens vêtus de noir attendajent. Milan Hlinka n'attendait plus. Il n'attendait plus depuis l'avant-veille. II y avait eu cette lourde journée noire, traversẻe par une certitude fulgurante: "lls nous ont lâchés! " Et puis le temps s'stait remis à couler, au petit bonheur, les jours ne se vivaient plus pour eux-mêmes, ga n'etait plus que des lendemains, il $n^{\prime} y$ aurait plus jamais que des lendemains.

A quinze heures trente, Mathieu attendait encore, au bord d'un horrible avenir; au même instant, à seize heures trente, Milan n'avait plus d'avenir. Le vieillard se leva, il traversa la pièce, les genoux raides, d'un pas noble et sautillant. Il dit : Messieurs! a et il sou*

\section{Première page du livre Le Sursis de Sartre}

cile à déceler et ne peut que faire l'objet d'une démonstrationjustification par la négative. En littérature, le montage, le changement ultra-rapide et pluriel de séries événementielles, et les brusques variations de focalisation n'existent que peu ou pas 
avant l'avènement du $7^{\mathrm{e}}$ art, avant le développement du langage cinématographique ${ }^{3}$. Il est permis de supposer que, par un effet rétroactif ${ }^{4}$, voire systémique, le cinéma, d’abord influencé par la narration littéraire, finit par servir de modèle pour celle-ci. Le véritable "montage littéraire», la juxtaposition de type "parataxique", discontinue en termes littéraires, de segments consacrés à des espaces-temps différents, souvent considéré comme l'apanage de l'avant-garde, devient caractéristique du roman seulement au $\mathrm{XX}^{\mathrm{e}}$ siècle.

Avec l'élaboration du montage qui, pour beaucoup de théoriciens, est le fondement même du langage cinématographique, c'est à la fois la représentation et la perception du monde en général qui changent, comme le confie Le Clézio:

Je pense que ce que le cinéma m'apporte, c'est moins une conception du roman ou de la narration et de ses techniques, qu'une conception du monde. Je veux dire que, me situant bien dans une époque où la culture est avant tout cinématographique, je vois et sens le monde cinématographiquement beaucoup plus que romanesquement ou poétiquement (p. 98).

L'élaboration de codes proprernent cinématographiques, dans laquelle la technicité a joué un rôle de tout premier ordre, a exercé sur les autres médias une stimulation créative. La représentation de la simultanéité, thème central du Sursis, est un bon exemple de l'apport esthétique du cinéma - comme la "conscience de la simultanéité", telle que nous la concevons aujourd'hui, ne serait-elle pas née avec le cinéma? - et montre aussi le caractère pionnier du roman de Sartre. Avec Le Sursis, Sartre a voulu révéler un moment crucial de la genèse de la Seconde Guerre mondiale, il a voulu montrer comment la guerre avait fini par s'imposer aux hommes, de manière simultanée, malgré (ou justement à cause de?) l'effroi qu'elle suscitait : «La guerre: chacun est libre et pourtant les jeux sont faits ${ }^{5}$ » (p. 1025).

Qu'est-ce que la simultanéité en littérature ou qu'est-ce que la représentation littéraire de la simultanéité? On peut essayer de répondre à cette question, très simplement, en opposant la simultanéité à la continuité. Deux "phénomènes" peuvent être considérés comme simultanés lorsqu’ils se produisent «au même 
moment" tout en étant séparés dans l'espace. Cependant, lorsqu'un lien causal les unit, ce n'est évidemment plus de simultanéité qu'il faudra parler, mais de continuité. Dans Le Sursis, on rencontre nombre de ces deux types de relation construite par le montage. Un exemple de simultanéité d'abord, choisi au tout début du roman ${ }^{6}:$ " $\AA$ quinze heures trente, Mathieu attendait encore, au bord d'un horrible avenir; au même instant, à seize heures trente, Milan n'avait plus d'avenir" (p. 734). Si la représentation de la simultanéité est ici quelque peu ironique, "quinze heures trente" et "seize heures trente" désignant le même "temps", elle n'en est pas moins "conventionnellement" explicite et manifeste, car signifiée par la locution «au même instant ${ }^{7}$ ". Mathieu et Milan étant séparés dans l'espace - l'un se trouve à Juan-les-Pins, l'autre à Prague —, il s'agit bien ici de simultanéité. La relation représentée peut être qualifiée de simultanéité simple, car évidente à travers la juxtaposition immédiate des deux termes qui sont, de plus, réunis par la locution «au même instant». À relever également le parallélisme provoqué par le mot "avenir" qui mérite l'appellation de transit: "Passage narrativement réglé d'un fragment de récit à un autre "(Bessalel et Gardies, p. 206).

Il n'y a pas que des simultanéités simples dans Le Sursis, mais également des simultanéités complexes, dont la relation suivante, entre la série événementielle consacrée au vieillard et celle consacrée à Milan ${ }^{8}$, est un exemple probant:

Le vieillard se leva, il traversa la pièce, les genoux raides, d'un pas noble et sautillant. Il dit : «Messieurs!" et il sourit affablement; il posa le document sur la table et en lissa les feuillets de son poing fermé; Milan s'était planté devant la table; le journal déplié couvrait toute la largeur de la toile cirée. Milan lut pour la septième fois: "Le Président de la République, et avec lui le Gouvernement n'ont rien pu faire quaccepter les propositions des deux grandes puissances, au sujet de la base d'une attitude future. Il ne nous restait rien d'autre à faire, puisque nous sommes restés seuls. "Nevile Henderson et Horace Wilson s'étaient approchés de la table, le vieillard se tourna vers eux, il avait l'air inoffensif et périmé, il dit: "Messieurs, voici ce qui nous reste à faire. "Milan pensait : "Il n'y avait rien d'autre à 
faire." Une rumeur confuse entrait par la fenêtre et

Milan pensait : "Nous sommes restés seuls" (p. 734).

La simultanéité est ici complexe car, quoique les deux termes se jouxtent, elle n'est explicitée par aucune marque conventionnelle du type "au même instant". Mais le lecteur a à sa disposition des transits et lit donc la simultanéité des actions du vieillard et de Milan sur la base de leur similitude — «se leva " et "s'était planté" - et grâce aux références communes à un texte: "le document" et "le journal». Un peu plus loin, le message que Milan lit et que le vieillard dit - en substance, "il n'y avait rien d'autre à faire" - , comme la pensée de Milan, "nous sommes restés seuls" - qui, elle, renvoie à l'article du journal — viennent renforcer cette lecture. Alors qu'il serait délicat de parler d'écriture cinématographique en ce qui concerne l'exemple précédent - avec focalisation sur Mathieu et Milan —, rien ne s'y oppose ici : c'est en effet grâce au montage alterné que le lecteur comprend ces séries événementielles comme simultanées, malgré l'absence de marque conventionnelle explicitant la simultanéité.

Si le cinéma n'a pas inventé la représentation de la simultanéité - on trouve déjà dans L'Odyssée un "montage " d'actions parallèles —, il a néanmoins fourni à l'artiste les moyens d'en révolutionner la représentation au niveau discursif afin de parvenir à une plus grande "illusion d'ubiquité ". Mais une fois appliquée à la littérature, cette écriture cinématographique, d'abord perçue comme continue dans le cours d'un film, peut être perçue comme discontinue au fil d'un texte littéraire. Cette discontinuité oblige à porter un intérêt à la réception, à intégrer, dans le processus créatif, la subjectivité du lecteur - le fameux "récepteur" de la théorie de la communication - qui, quittant son rôle passif, devient un "lecteur productif ${ }^{10}$ ". Dans le champ des études littéraires, la production de sens est désormais considérée comme une tâche incombant autant au récepteur qu'à l'émetteur du message artistique; les théoriciens, qui ont étudié cette question, ont ainsi déplacé la définition du texte: de "produit fini", il est devenu interface " générateur de diégèse entre l'auteur et le lecteur.

Ce n'est pas un hasard si Sartre utilise une technique narrative inspirée du film, car il partage avec les structuralistes et autres 
néo-formalistes iqui, faut-il le rappeler, ont jeté, avec la critique d'inspiration psychanalytique, les bases de la sémiologie du cinéma) une certaine conception philosophique du personnage. Comme l'écrit le narratologue américain Seymour B. Chatman,

[...] narrative theory, they (formalists and structuralists) say, must avoid psychological essences; aspects of character can only be "functions". They wish to analyse only what characters do in a story, not what they are - that is, "are" by some outside psychological or moral measure (p. 111).

Se concentrer sur l'existence et non sur l'essence des personnages, voilà un principe constitutif que partagent le cinéma et l'existentialisme: "Le film est toujours davantage behavioriste: il montre plus aisément des comportements qu'une intériorité" (Aumont et Marie, p. 108) ${ }^{12}$.

Ce n'est que sur le plan de l'expression, au niveau du discours, qu'il est possible de penser la narration littéraire en rapport avec la narration cinématographique. Je suis partiellement d'accord avec Michel Contat lorsqu'il écrit que
[...] le montage du Sursis évoque celui de la télévision en régie directe. Sartre se trouve, par rapport à la matière romanesque qu'il brasse, dans la position du réalisateur qui a devant lui une multitude d'écrans lui donnant l'image captée en direct et en continuité par des caméras dispersées aux quatre coins de l'espace (ici l'Europe) et qui choisit parmi toutes ces images, en passant d'un écran à un autre, celle qu'il va transmettre sur l'écranı unique des récepteurs (p. 1967).

Pour ce qui est des enchaînements de segments simultanés qui ne sont pas agencés discursivement par une opération de transit, la comparaison de Contat avec la télévision est pertinente. Elle ne l'est cependant plus lorsqu'il s'agit de l'articulation concertée des segments par le biais de transits et de raccords; celle-ci ne saurait en aucun cas participer, à la base, de l'esthétique de la télévision "en régie directe» qui est très différente, comme l'explique très clairement Youssef Ishaghpour, de l'esthétique du cinéma ${ }^{13}$. Comme l'indique Contat lui-même, 
c'est «[...] le montage cinématographique, qui met bout à bout des plans déjà découpés" (p. 1967), qui a inspiré Sartre pour Le Sursis. Car, dans la très grande majorité des cas, les segments simultanés sont agencés par des transits, en général des "pivots scripturaux ${ }^{14}$ " qui se confondent parfois avec des raccords et qui visent à atteindre une certaine cohérence narrative. Lorsque Sartre articule des segments selon la logique de la continuité ${ }^{15}$, il utilise des raccords, notamment dans la séquence avec focalisation sur Charles et sur l'infirmière que nous analyserons plus loin. Ces raccords, censés assurer une impression de fluidité, sont absents de toute émission télévisée en régie directe, la simultanéité des événements et de leur narration ne permettant que rarement le choix des raccords - et des transits par la même occasion.

De plus, il ne faut pas oublier que le cinéma est l'aîné de la télévision, par conséquent, pour ce qui est du montage, c'est la petite sœur qui a bénéficié des expériences du grand frère: le cinéma était là d'abord et c'est lui qui a servi de substrat à la télévision. Il ne faut pas perdre de vue non plus qu'à l'époque de la genèse du Sursis, au début des années quarante, la télévision n'en est qu'à ses balbutiements, alors que le cinéma, lui, est déjà riche de près d'un demi-siècle d'expérience. Je ne crois donc pas qu'il soit judicieux de dire que "le montage du Sursis évoque celui de la télévision en régie directe"; outre son écriture cinématographique, il faut plutôt rappeler le caractère prospectif ou pionnier du roman de Sartre, préfigurant d'une certaine manière l'esthétique future de la télévision - ainsi que des nouveaux médias - et témoignant du «[...] sens qu'il [Sartre] eut du monde, de l'éclatement des frontières, de l'internationalisation de tout" (Brochier, 1985, p. 9).

Postuler l'écriture cinématographique d'un roman signifie avant tout attirer l'attention sur le plan de l'expression, sur le récit et non sur l'histoire. "L'organisation du texte entier en constituants textuels immmédiats est appelée macrostructure. L'organisation de la plus petite unité textuelle, le microtexte, en phrases constitue la microstructure (Lerot, p. 135). Ce découpage textuel est très approprié à la structure du Sursis; celui-ci s'étend sur 401 pages et est divisé en huit chapitres, chaque cha- 
pitre correspondant plus ou moins à une journée de la semaine des accords de Munich, en septembre 1938. À l'intérieur de cette répartition apparente en chapitres, Le Sursis est doublement structuré par une macrostructure et une microstructure. La macrostructure comprend 33 syntagmes. Par syntagme ${ }^{16}$, j'entends l'ensemble des segments compris entre deux indices temporels signifiant une progression explicite sur l'axe chronologique $^{17}$ et qui correspond à l'unité minimale de la macrostructure, au sein de laquelle s'articule la microstructure, c'est-à-dire les segments. Par segment ${ }^{18}$, j'entends l'unité microstructurale "[...] qui se caractérise par son autonomie de sens et de configuration" (Bessalel et Gardies, p. 30), par son articulation autour de deux variables (personnages et lieux) correspondant à l'unité minimale de la microstructure. La macrostructure équivaut donc à l'articulation des syntagmes et, du point de vue narratif, elle sert à expliciter la progression chronologique du texte. Le deuxième syntagme commence temporellement après le premier et se termine avant le troisième, et ainsi de suite. Dans $L e$ Sursis, l'arrangement discursif des syntagmes n'a, en soi, rien de cinématographique puisque les transitions entre eux sont marquées conventionnellement. Le troisième syntagme, par exemple, débute par "Dix-huit heures dix à Godesberg" (p. 753); le lecteur a donc directement connaissance de la progression du récit par l'indice temporel "dix-huit heures dix" qui indique clairement la démarcation par rapport à l'indice temporel précédent, "dix-sept heures trente à Berlin, seize heures trente à Paris (p. 739). Pourquoi prendre appui sur la chronologie pour "découper" le roman? Parce que, dans la perspective d'une lecture axée sur la simultanéité, seuls les indices temporels peuvent être pris en considération pour révéler la progression du roman, la notion de simultanéité pouvant être réduite à la formule: espaces = variables / temps = invariant. Il faut cependant remarquer que les marques énoncées du passage du temps - toute référence au jour et à l'heure - sont à la fois des invariants et des variables: invariants parce que, à l'intérieur d'un même syntagme, ils sont l'élément constant qui relie les différentes séries événementielles parallèles entre elles, c'est-à-dire les segments; variables au niveau de l'articulation des syntagmes puisqu'il y a 
nécessairement entre chacun d'eux une évolution chronologique.

C'est à propos de la microstructure du Sursis qu'il devient légitime de parler d'écriture cinématographique puisque le très grand nombre de segments formant les différentes séries événementielles "éclatées" s'articulent le plus souvent sans marques conventionnelles du temps et, à fortiori, du mouvement du temps: les segments sont articulés selon une logique de montage cinématographique qui peut se permettre d'omettre les transitions (marquées) sans que la compréhension narrative du lecteur n'en souffre ${ }^{19}$. Aujourd'hui, en effet, cette simple juxtaposition est devenue la norme: “[...] das bescheidene Prinzip reinster Addition gilt als völlig zulänglich. Normal ist heute die Simultanlieferung völlig disparater Elemente» (Anders, p. 141).

À la manière des journaux intimes et des chroniques, Le Sursis commence par une date, "Vendredi 23 septembre", servant de titre au premier chapitre. Dès le début, le lecteur est donc plongé dans l'historicité. Après cet ancrage sur l'axe de la temporalité commence le texte romanesque qui, lui aussi, nous plonge dans un moment: "Seize heures trente à Berlin, quinze heures trente à Londres» (p. 733). Aux indices temporels sont venus s'ajouter deux indices spatiaux, "à Berlin" et "à Londres"; d'emblée, nous sommes confrontés au thème central du second tome des Chemins de la liberté: la représentation de la simultanéité qui, sur le plan de l'expression, se distingue par l'enchevêtrement d'un grand nombre de séries événementielles, sans transitions explicites ni marques conventionnelles de la simultanéité (simultanéité complexe). La phrase incipit pose d'entrée de jeu l'ubiquité de l'instance narrative qui, à l'image d'Asmodée, va se promener en toute liberté dans l'espace-temps diégétique. L'usage de la simultanéité est ici chargée d'ironie car, bien qu'il s'agisse du même moment, deux indices temporels sont nécessaires - «seize heures trente» et "quinze heures trente»puisque des fuseaux horaires différents séparent Londres de Berlin. Ceci réclame une attention particulière de l'instance de réception. Contrairement à l'exemple commenté ci-dessus Mathieu à 15 h 30 et Milan à 16 h 30 -, la simultanéité n'est pas ici «lisible» à travers les seuls indices temporels, elle n'est pas 
explicitée par une marque conventionnelle; c'est au lecteur qu'incombe le travail de découpage, le «déchiffrage du montage $^{20} »$. Ce n'est qu'à cette condition que la fonction d'invariant du temps, l'impératif de toute relation de simultanéité, pourra pleinement jouer son rôle: quand le lecteur aura reconnu que "seize heures trente" et "quinze heures trente" ont le même référent, il sera alors légitime de parler de simultanéité.

Ensuite, de ce niveau assez général - les métropoles -, on passe à un niveau plus "personnalisé» par la présence, dans la deuxième phrase, d'un protagoniste: "L'hôtel s'ennuyait sur sa colline, désert et solennel, avec un vieillard dedans» (p. 733). La seconde phrase contient elle aussi des indices spatiaux: "l'hôtel sur sa colline". La question que, suivant une logique associative, le lecteur est en droit de se poser est la suivante: "Cet hôtel, qui se trouve sur une colline, est-il situé à Berlin ou à Londres?» Ou s'agirait-il d'un troisième lieu diégétique? Sans nous apporter de réponse, la troisième phrase introduit plusieurs nouveaux éléments: "À Angoulême, à Marseille, à Gand, à Douvres, ils pensaient: "Que fait-il? Il est plus de trois heures, pourquoi ne descend-il pas?" (p. 733). Quatre nouveaux toponymes: Angoulême, Marseille, Gand et Douvres, et un nouveau sujet grammatical, le pronom personnel «ils»; un temps grammatical, l'imparfait et, via le discours direct, une référence intradiégétique à un personnage, «il», ainsi qu'un nouvel indice temporel, «plus de trois heures». Ce segment, qui s'articule autour du pronom personnel "ils" - ici le sujet grammatical - est, à l'écrit, quelque peu flou du point de vue référentiel: qui sont« ils»? Le texte littéraire ne possédant pas le pouvoir de monstration du cinéma, le lecteur doit accepter l'identité incertaine des personnages et se contenter, dans un premier temps, de réponses potentielles avec, pour seule certitude : «ils», à la forme du pluriel, pensent à un autre "ill", au singulier. Le montage de tels segments justifie la critique de Geneviève Idt qui, à propos de l'écriture du Sursis, parle de "foisonnement anarchique» et de "jeu étourdissant" (p. 87-88). Critique à laquelle on pourrait ajouter le reproche d'une écriture "floue» - «[...] von dem sich nicht mit Sicherheit sagen läßt, ob er Kommentar des Autors oder der Figur ist " (Kaemmerling, p. 193). Le passage de 
la focalisation zéro à la focalisation interne est dû au changement de type de discours : «ils» savent qui «il» est parce que d'indirect, le discours se fait direct, la perspective devenant ainsi interne.

Il était assis dans le salon aux persiennes demi-closes, les yeux fixes sous ses épais sourcils, la bouche légèrement ouverte, comme s'il se rappelait un souvenir très ancien. Il ne lisait plus, sa vieille main tavelée, qui tenait encore les feuillets, pendait le long de ses genoux. Il se tourna vers Horace Wilson et demanda: "Quelle heure est-il?" et Horace Wilson dit: "Quatre heures et demie, à peu près." Le vieillard leva ses gros yeux, eut un petit rire aimable et dit: "Il fait chaud" (p. 733).

Dans ce nouveau segment, nous avons affaire à un changement d'espace, et l'instance narrative revient alors à un personnage, «il», qui se trouve inséré dans un contexte toponymique, "dans le salon aux persiennes demi-closes". Comme ce changement d'espace a lieu sans référence à un changement de temps, on peut parler d'ubiquité et partant, de simultanéité; l'expression de celle-ci, n'étant pas explicitée par une marque conventionnelle, peut s'apparenter à la narration cinématographique qui n'hésite pas à juxtaposer en parataxe des segments consacrés à diverses séries événementielles.

Ce nouveau segment introduit également un nouveau personnage, figure historique cette fois: Horace Wilson. Le "conseiller industricl du gouvernement anglais" (Contat, p. 1972) est donc l'allocutaire du pronom personnel «il ", qui va se révéler être le vieillard de la deuxième phrase. Dernière information à relever dans ce segment: l'indice temporel "Quatre heures et demie, à peu près" qui signifie une fois de plus la simultanéité des segments. Passage ensuite à un segment très général, qui s'actualise dans la référence à un toponyme "général ", "l'Europe ", et à un objet de focalisation lui aussi "général " et abstrait, "les gens": "Une chaleur rousse, crépitante, pailletée s'était affalée sur l'Europe; les gens avaient de la chaleur sur les mains, au fond des yeux, dans les bronches; ils attendaient, écœurés de chaleur, de poussière et d'angoisse" (p. 733). Le mot 
"chaleur" et ses implications au niveau de l'histoire renvoient à l'assertion énoncée par le vieillard dans le segment précédent, "Il fait chaud", et opère donc un transit d'ordre sémantique. Le segment général, cette fois davantage récit écrit que récit filmique - imaginons la difficulté de représenter à l'écran cette chaleur sur toute l'Europe en un temps discursif aussi réduit —, fait appel à un montage qui repose sur l'association, voire la causalité. Dans l'esprit du lecteur, la "souffrance» des gens est due à la canicule, le plan très général et abstrait de l'Europe se concrétisant ainsi dans la personne des "gens".

Suivent ensuite cinq segments consacrés: 1) aux journalistes, 2) aux trois chauffeurs, 3) à de longs Prussiens vêtus de noir, 4) à Milan Hlinka, 5) à Mathieu, qui, contenant chacun le verbe "attendre" conjugué à l'imparfait, peuvent être qualifiés de segments parallèles.

Dans le hall de l'hôtel, les journalistes attendaient.

Dans la cour, trois chauffeurs attendaient, immobiles au volant de leurs autos; de l'autre côté du Rhin, immobiles dans le hall de l'hôtel Dreesen, de longs Prussiens vêtus de noir attendaient. Milan Hlinka n'attendait plus. Il n'attendait plus depuis l'avant-veille. [...] À quinze heures trente, Mathieu attendait encore (p. 733-734).

Cette série est typiquement filmique dans la mesure où elle induit dans l'esprit du lecteur le parallélisme des "attentes" par la simple juxtaposition des segments, pourtant dépourvus de toute référence explicite à la simultanéité. Les locutions adverbiales ou prépositives telles que "au même moment" ou "et pendant que " erc., marques conventionnelles, en lesquelles prend corps la notion de simultanéité, absentes du texte écrit, sont reçues comme implicites par le lecteur qui, par sa connaissance du langage cinématographique, lit ces différents segments juxtaposés comme parallèles dans le temps - il serait évidemment intéressant de savoir si un lecteur d'une quelconque époque préfilmique serait en mesure de lire cette simultanéité ${ }^{21}$, mais cela me paraît quelque peu difficile à vérifier de manière vraiment empirique. Le cinéma, par sa nécessité ontologique de "raccordement bout à bout de plans", par sa syntaxe, 
transcende la linéarité pour mieux révéler le "non-montré" et produire une plus-value sémantique par le montage. Souvenonsnous de la fameuse phrase de Sergeï Eisenstein: "La juxtaposition de deux fragments de films distincts, mis bout à bout, s'apparente moins à leur somme qu'à leur produit» (p. 215). Ici, la somme de l'assemblage des fragments est en position de déficit cognitif par rapport à son produit; la somme peut être formulée comme "l'attente de plusieurs protagonistes", alors que son produit contient une information supplémentaire et prégnante, la simultanéité: "l'attente de plusieurs protagonistes en même temps (en des endroits différents, bien sûr)".

Ces quelques segments formant juste un peu plus de la première page du roman contiennent déjà en substance l'ensemble des caractéristiques discursives du Sursis.

Très souvent, comme nous l'avons déjà vu dans l'analyse des premiers segments du roman, Sartre écrit la simultanéité en l'appuyant sur des transits, comme il le fera pour le scénario du film Les jeux sont faits. Le "contemporain capital" (Brochier, 1996, p. 72) y utilise le même type de transits entre les plans que pour l'articulation de nombreux segments dans Le Sursis:

La chambre d'Ëve: "Elle appelle [...] :- Lucette! Lucette!

La rue des conspirateurs: Un jeune homme [...] appelle: — Pierre! Pierre! (p. 16-17).

Les unités filmiques sont mises en parallèle par la reprise, dans le plan avec focalisation sur le jeune homme, du verbe "appelle", qui renvoie au plan précédent dans lequel l'action est également celle d' "appeler". On trouve ce procédé de transit à maintes reprises dans Le Sursis. Dans l'extrait suivant du roman, c'est surtout le verbe "dire" qui assure la fonction de pivot scriptural avec d'autres éléments, dont des actes de langage ${ }^{22}$ (chanter, appeler, etc.) :

Au cinquième étage de l'hôtel Massilia, une femme qui prenait le frais à son balcon l'entendit, elle dit: "Gomez! viens écouter le nègre, c'est charmant!" Milan pensa à sa jambe et sa joie s'éteignit, il serra fortement l'épaule d'Anna et dit: “Ils ne voudront pas de 


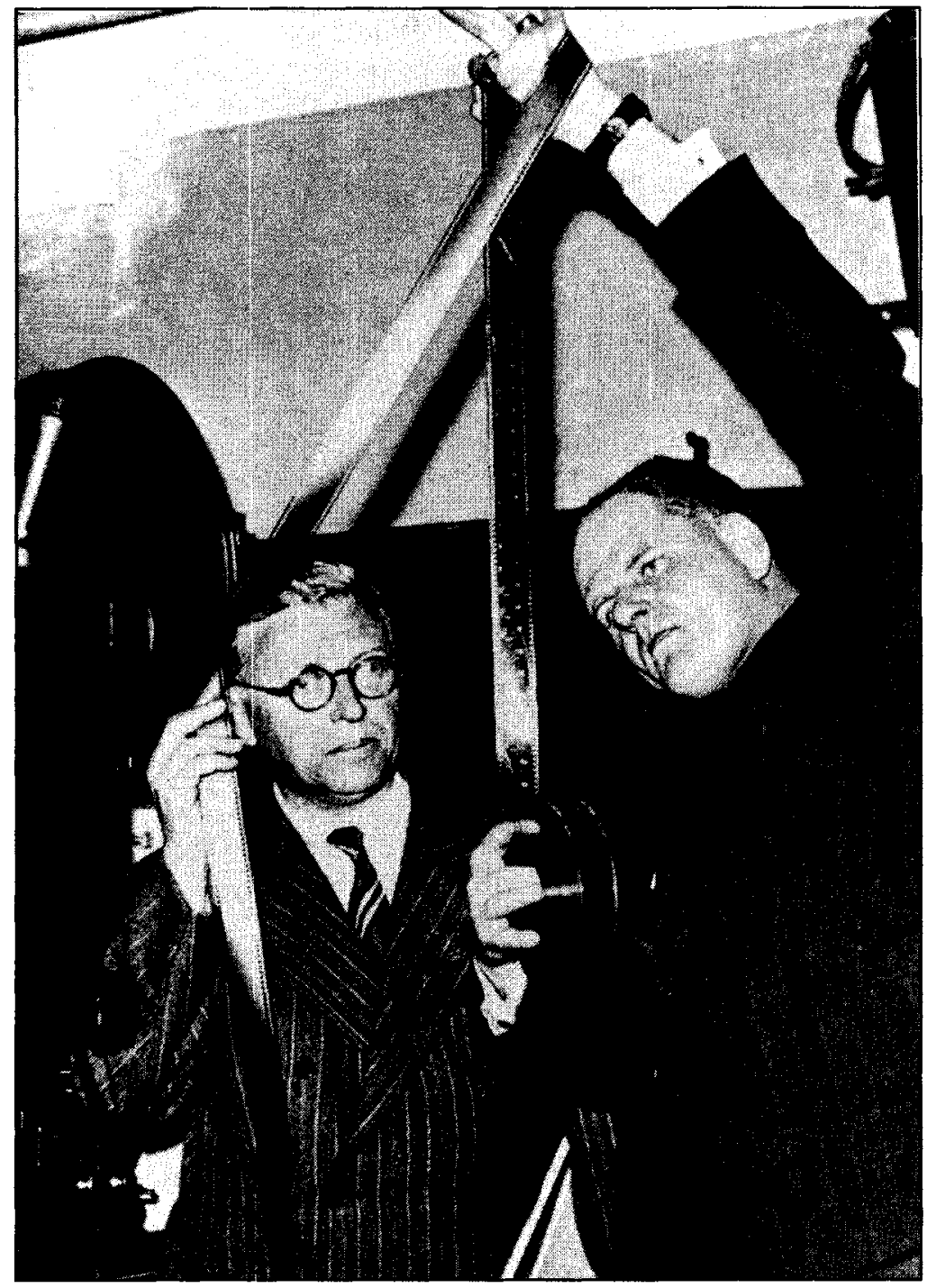

\section{Sartre examine avec Delannoy les premières pellicules du film Les jeux sont foifs.}

moi, je ne suis plus bon à rien. " Et le nègre chantait. Armand Viguier était mort, ses deux mains pâles s'allongeaient sur le drap, les deux femmes le veillaient en causant des événements, elles avaient sympathisé tout de suite, Jeannine prit une serviette éponge et s'essuya les mains, puis elle se mit à lui frotter les cuisses, 
Chamberlain disait: «En ce qui concerne le premier paragraphe, je présenterai deux objections " et le nègre chantait: "Bei mir bist du schön; cela signifie: "Vous êtes pour moi la plus jolie"."

Deux femmes s'arrêtèrent, il les connaissait, Anina et Dolorès, deux putains de la rue du Lacydon, Anina lui dit: "Té! tu chantes?" et il ne répondit pas, il chantait et les femmes lui sourirent et Sarah appela avec impatience: "Gomez, Pablo venez donc! qu'est-ce que vous faites? Il y a un nègre qui chante, c'est charmant " (p. 794).

Sartre articule ici ces 11 segments par un système complexe de transits, qui va bien au-delà de la simple "reprise» d'un élément d'un segment à l'autre. Chaque segment - à l'exception de celui consacré à Armand Viguier et aux deux femmes, et de celui consacré à Jeannine, qui sont suturés différemment contient un verbe qui engage la parole ou la sous-entend, il contient un acte de langage: Hitler qui va prononcer son discours, la voix qui «mont[e] dans l'air pur", le verbum dicendi "dire" (quatre occurrences), le verbe "chanter" (trois occurrences) et enfin le verbe "appeler». Les deux premiers segments entretiennent un rapport de continuité par la causalité, ou plutôt de pseudo-causalité puisqu'il s'agit de deux séries événementielles différentes: alors que dans le premier segment un personnage va entamer un discours, l'instance narrative fait référence, dans le segment suivant, à une voix. Le troisième segment est raccordé au précédent suivant le même principe de cause à effet, un personnage, Anna, parlant de cette même voix qui chante. Le quatrième segment contient le même verbe, "dit", que le segment précédent. Ensuite, le cinquième segment met en évidence la simultanéité des segments précédents, puisque c'est toujours du "nègre [qui] chantait" dont il s'agit, et fait par la même occasion référence au segment précédent. L'opération de transit entre les deux segments suivants, le sixième et le septième, est moins évidente mais tout aussi efficace: le segment consacré aux deux femmes est suturé au précédent par le verbe "causer» et celui consacré à Jeannine l'est au précédent par la référence aux "mains ${ }^{23}$ ". Le huitième segment est «transité» explicitement au segment précédent avec focalisation sur Milan par la présence 
du verbe "dire", implicitement au sixième segment par le parallélisme entre "dire" et "causer». Le neuvième segment, consacré au nègre, est suturé au précédent par le verbe "chanter" qui renvoie au verbum dicendi "disait", signifiant toujours la simultanéité des autres actions. Le dixième segment est en rapport avec le neuvième du point de vue du contenu, puisqu'il y est question du nègre qui chante, et renvoie au huitième segment par le verbe « dire». Le dernier segment du syntagme, le onzième, s'aligne sur le précédent par le verbe "appeler" qui fait référence à "dire", ainsi que par son contenu: le nègre qui chante.

Cependant, dans Le Sursis, les connexions opérées entre les séries événementielles ne sont pas toujours explicites; le plus souvent, la simultanéité est plus complexe, car elle n’est ni introduite par une marque conventionnelle, ni induite par un pivot scriptural, mais par leur simple juxtaposition. Il en est ainsi dans le passage suivant - syntagme 24 , situé juste avant le chapitre «27 septembre»:

Il était plus de minuit, le Sportpalast était obscur et désert, chaises renversées, bouts de cigares écrasés, M. Chamberlain parlait à la radio, Mathieu errait sur le quai du Vieux-Port, en pensant: "C'est une maladie, tout juste une maladie; elle est tombée sur moi par hasard, elle ne me concerne pas, il faut la traiter par le stö̈cisme comme la goutte ou les maux de dents." M. Chamberlain dit :

"J'espère que le chancelier ne rejettera pas cette proposition qui est faite dans le même esprit d'amitié avec lequel j'ai été accueilli en Allemagne, et qui, si elle est acceptée, satisfera le désir allemand de l'union des Sudètes avec le Reich, sans verser de sang en aucune partie de l'Europe."

Il fit un geste de la main pour indiquer qu'il avait terminé et s'éloigna du micro. Zézette, qui ne pouvait s'endormir, s'était mise à la fenêtre et regardait les étoiles au-dessus des toits, Germain Chabot ôtait son pantalon dans le cabinet de toilette. Boris attendait Lola dans le hall du casino; partout, dans les airs, inécoutée ou presque, une fleur sombre tentait d'éclore: If the moon turns green, interprété par le jazz de l'hôtel Astoria et retransmis par Daventry (p. 1035). 
Il s’agit ici de six séries événementielles: 1) le Sportpalast où Hitler a tenu son discours, 2) Chamberlain, 3) Mathieu, 4) Zézette, 5) Germain Chabot et 6) Boris et Lola. Celles-ci sont mêlées par des phrases qui, vu leur portée ubiquitaire, ont ici une fonction "encadrante», elles servent à mettre en évidence la simultanéité des divers segments, sept au total - deux étant consacrés à Chamberlain: "Il était plus de minuit" et "partout, dans les airs, inécoutée ou presque, une fleur sombre tentait d'éclore: If the moon turns green, interprété par le jazz de l'hôtel Astoria et retransmis par Daventry. "Il n'y a vraiment alternance qu'au niveau de l'articulation des trois premiers segments, selon le schéma Chamberlain-Mathieu-Chamberlain; la simultanéité est ici, par le fait même de l'alternance, tout à fait manifeste. Malgré la ponctuation «franche», c'est-à-dire l'utilisation du point, et contrairement à celle plus "douce" utilisée entre les deux premiers segments, une virgule, les segments se lisent comme simultanés; d'une part grâce aux "phrases encadrantes" et d'autre part, ce qui est plus important car plus cinématographique, grâce au montage de type parataxique que, dès le début du roman, Sartre a érigé en système d'écriture et que le lecteur du Sursis s'est vu imposer comme grille de lecture.

Le montage des segments à la suite de la scène de masturbation de Charles par l'infirmière - vers la fin de la journée " 23 septembre" - représente un exemple type d'entrelacement des séries événementielles avec continuité par raccord sémantique.

Il était étendu, les mains sous la nuque, il se sentait tout gris, il dit: "On l'aime bien sa petite poupée." Et elle tressaillit, elle dit: "Oui..." Comme chaque soir, elle avait peur. "Oui, je vous aime bien!" Des fois elle acceptait, des fois elle disait non, mais ce soir elle n'oserait pas. "Alors on lui fait sa perite caresse, sa petite caresse du soir?» Elle soupira, elle était tout honteuse, c'était amusant. Elle dit: "Pas ce soir." Il souffla un peu, il dit: «Pauvre petite poupée elle est si agitée, cela lui ferait tant de bien. Pour la faire dormir, vous ne voulez pas? Non, vous ne voulez pas? Tu sais bien, ça me calme toujours..." Elle prit son visage d'infirmièremajor, comme lorsqu'elle le mettait sur le bassin, sa tête 
devint toute raide sur ses épaules, elle ne fermait pas les yeux, mais c'était tout comme si elle s'arrangeait pour ne rien voir et ses mains, par en-dessous, le déboutonnèrent prestement, des mains de spécialiste, et son visage qui était si triste, c'était très amusant, la main entra, si douce, une pâte d'amandes, Odette sursauta et dit: "Vous m'avez fait peur: est-ce que Jacques est avec vous?" Charles soupira, Mathieu dit non (p. 790-791).

Le segment avec focalisation sur Charles et l'infirmière se termine par le geste de celle-ci: "la main entra, si douce, une pâte d'amandes». Ensuite, par un faux montage en continuité, le narrateur focalise sur Odette: celle-ci «sursauta et dit: "Vous m'avez fait peur: est-ce que Jacques est avec vous?" ". Le raccord entre ces deux segments peut être qualifié d'associatif, la réaction d'Odette nous paraissant légitime, dans la mesure où elle est la réponse à un stimulus énoncé. Cependant ce stimulus est énoncé dans le segment précédent, qui ne focalise pas sur elle: c'est donc au lecteur d'opérer la connexion sémantique entre ces deux segments - Wolfgang Iser parle à ce propos de Leerstellen. Ici, la narration peut être qualifiée de cinématographique, car elle est avant tout visuelle: le changement d'objet de la focalisation, de Charles et l'infirmière à Odette, n'est que difficilement repérable à l'écrit puisqu'il n'est pas explicité, alors que s'il s'agissait d'un film, il serait très probablement reconnu immédiatement, puisque l'objet de la focalisation - Charles et l'infirmière, ensuite Odette - serait visible sur l'écran (à moins qu'il ne s'agisse d'un très gros plan ne permettant pas la reconnaissance immédiate).

Le segment suivant s'inscrit dans un rapport de rupture avec le précédent, mais de continuité avec le segment précédent consacré à Charles, son soupir étant la réaction au geste de l'infirmière. Puis, retour à Odette, en position d'allocutaire dans le segment suivant - "Mathieu dit non" - qui est syntaxiquement suturé au segment précédent par une virgule. La négation "non" est l'élément sémantique qui assure le transit avec le segment suivant puisque Maurice la répète. Tous les raccords que Sartre utilise ici dans son montage en (fausse) continuité sont un pur produit de la narration cinématographique, dans la 
mesure où celle-ci s'est spécialisée dans une logique de causalité, caractéristique du cinéma classique hollywoodien. Or, il s'agit ici de séries événementielles différentes qui se déroulent dans un même temps, mais dans des espaces différents. Aucun lien causal ne les reliant, il ne peut par conséquent s'agir de causalité. Néanmoins, Sartre utilise ici des raccords qui, quoique faux puisque suturant des séries événementielles différentes, induisent à la lecture une "illusion de continuité». Il faut donc y « regarder» à deux fois pour déconstruire le texte et pour reconnaître la nature des relations - simultanéité ou continuité qu'entretiennent entre eux les segments, ce qui n'est pas chose aisée, Sartre ayant choisi une technique narrative très sophistiquée qui mêle continuité et simultanéité.

Les premiers segments du cinquième syntagme - début du second chapitre, "Samedi 24 septembre" - nous livrent un bel exemple de la difficulté occasionnelle de distribution des segments et du présupposé d'une lecture cinématographique du roman, la confusion étant en grande partie nourrie par le parallélisme des situations induit par les pivots scripturaux - ici, le verbe "penser" - ainsi que le champ sémantique abordé - le sommeil :
À Crévilly, sur le coup de six heures, le père Croulard entra dans la gendarmerie et frappa à la porte du bureau. Il pensait: "Ils m’ont réveillé. "Il pensait qu’il leur dirait : "Pourquoi qu'on m'a réveillé ?" Hitler dor- mait, Chamberlain dormait, son nez faisait une petite musique de fifre, Daniel s'était assis sur son lit, ruisse- lant de sueur, il pensait: "Ça n'était qu'un cauchemar!" (p. 795).

Le quatrième segment, "son nez faisait une petite musique de fifre", ne pose aucun problème de distribution si on se contente d'accepter la linéarité comme constitutive. Or, il s'agit ici d'un roman "monté", dans lequel l'ordre de la perception humaine n'est pas la règle. Envisagé dans une relation anaphorique à travers l'adjectif possessif "son", ce segment peut alors tout aussi bien renvoyer à Hitler, à Chamberlain ou encore au père Croulard. Envisagé dans une relation cataphorique, toujours à travers l'adjectif possessif "son", ce segment peut renvoyer au segment 
suivant, qui focalise sur le personnage de Daniel. Il est donc presque impossible pour le lecteur du Sursis de décider avec certitude de quel nez il s'agit. On peut interpréter ce déficit cognitif comme ceci : Sartre a écrit filmiquement, c'est-à-dire "audiovisuellement ", la distribution du segment ne posant alors aucun problème... ni à ses yeux ni à ses oreilles. Geneviève Idt suggère à raison qu'il s'agit ici d'une concrétisation du concept philosophique sartrien de "l'universel singulier» (Sartre, 1972), cette "petite musique de fifre" pouvant être attribuable à chacun des personnages. Si je reconnais la justesse du propos, je ne peux cependant m'empêcher de penser que le plan de l'expression perturbe ici le lecteur et l'invite à "déconstruire" le texte pour en interroger les rouages. Car ce n'est qu'après avoir compris la mécanique narrative sous-tendant le récit que le lecteur trouvera le chemin de l'explication philosophique, compte tenu que «[...] der Verstand beherrscht, kontrolliert, während man bei der Wahrnehmung von der Wirklichkeit überrumpelt wird" (Koenot, p. 142).

Les segments alternés consacrés aux déboires de Gros-Louis qui cherche son nègre et au sentiment de nausée de Pierre sur le bateau illustrent l'ambiguïté qui peut naître d'un montage reposant sur des procédés endophoriques et coréférentiels.

Mario avait pris Gros-Louis par la taille, le capitaine avait saisi la combinaison par une bretelle, Maud ne put s'empêcher de rire: "Mais vous la tenez à l'envers!" Mario se penchait en avant, il serrait fortement la taille de Gros-Louis et se frotrait la tête contre son estomac, il disait: "T'es mon pote, pas vrai Starace, c'est mon petit pote, on s'aime, nous deux." Et Starace riait en silence, sá tête tournait, tournait, tournait, ses dents brillaient, c'était un cauchemar, sa tête était toute bruissante de cris et de lumières, il s'en allait vers d'autres bruits et d'autres lumières, ils ne le lâcheraient pas de la nuit, le rire de Starace, son visage brun qui montait et descendait, la petite gueule de fouine de Mario, il avait envie de vomir, la mer montait et descendait dans l'estomac de Pierre, il savait très bien qu'il ne retrouverait jamais son nègre, Mario le poussait, Starace le tirait, le nègre était un ange et moi je suis en enfer (p. 882-883). 
Le "fourmillement polarisé" (Bory, p. 81) auquel le lecteur est confronté ici réclame un découpage réfléchi. Une fois de plus dans Le Sursis, le contenu et la forme sont en accord: on retrouve le sentiment de nausée et d'étourdissement des personnages au niveau de l'arrangement discursif du roman. Quelle est la tête qui "tournait, tournait, tournait [...] toute bruissante de cris et de lumières", quel est le «visage brun qui montait et descendait"? Qui pense: "Et moi je suis en enfer"? L'enchaînement des propositions - qui correspond à l'enchaînement des segments - obéissant à des règles de (fausse) continuité - car articulant des séries événementielles différentes -., le segment "il avait envie de vomir" ne peut être distribué avec certitude, dans un premier temps, ni à Gros-Louis (rapport anaphorique) ni à Pierre (rapport cataphorique). Le contexte, qui généralement fournit les informations nécessaires à la clarification d'une situation confuse, est lui-même source d'ambiguïté. Le pronom personnel "il " n'a pas ici valeur distinctive et ne permet pas, une fois de plus, de trouver dans le texte les informations pertinentes permettant d'élucider les questions que l'œuvre génère dans l'esprit du lecteur. Car il est question d'un estomac et d'une "tête [qui] tournait, tournait, tournait [...] toute bruissante de cris et de lumières " dans les segments à focalisation sur Mario et GrosLouis, mais également de "la mer [qui] montait et descendait dans l'estomac de Pierre». Qui a donc la nausée? Et qui, comme nous l'apprenons à travers le changement de focalisation, pense: «Et moi je suis en enfer»? Vu les transits topiques - le sentiment de malaise - qui assurent le parallélisme entre les différentes séries événementielles, il est presque impossible d'en déterminer le référent. Le montage est ici cinématographique, dans la mesure où Sartre juxtapose des espaces sans les situer précisément (comme le mode d'écriture classique le prévoit) à l'aide de marqueurs linguistiques assurant le changement d'espace. Ici, Sartre écrit visuellement. Si ces segments étaient des segments d'un film, chacun d'entre eux serait identifiable l'effet de réel au cinéma, l'analogie visuelle et sonore avec notre monde favorisant l'identification ${ }^{24}$ - et non plus "flou" comme c'est le cas ici. La polysémie née du montage des segments engendre la concrétisation de l'universel singulier. 
Dans le syntagme 32 - qui correspond à la "Nuit du 29 au 30 septembre» (p. 1118 à 1825) - , sur un temps discursif correspondant à sept pages, Sartre met en parallèle le viol d'Ivich et les pourparlers sur la Tchécoslovaquie. Pour ce faire, il utilise le procédé du montage alterné, consacrant 10 segments aux faits historiques et neuf à l'événement fictif. Dans l'ensemble, les segments sont, d'un point de vue discursif, de même longueur, à l'exception de ceux consacrés à la lecture des accords par Mastny, qui sont sensiblement plus longs. La structure de l'alternance entre la séquence des pourparlers réunissant Masaryk et Mastny, Sir Horace Wilson, Chamberlain et Ashton-Gwatkin, Daladier et Léger, et la séquence du viol d'Ivich, induit à la lecture une relation métaphorique entre l'Histoire et l'histoire. Pour s'assurer de la bonne réception de l'analogie qu'il a choisi d'établir, Sartre fait en sorte que «[...] le rythme de l'alternance ne soit pas trop lent” (Metz, p. 106).

Dans Le Sursis, Sartre monte donc des espaces-temps concrets, centrés sur une série événementielle à la fois. Il aurait pu très bien adopter une perspective plus générale, par la focalisation zéro ${ }^{25}$, et montrer l'inquiétude, par exemple, en adoptant comme système textuel une écriture "surplombante», ce qu'il ne fait que rarement et ce qui produit un résultat bien moins "monté", comme en témoigne l'exemple suivant: "Les gens avaient de la chaleur sur les mains, au fond des yeux, dans les bronches; ils attendaient, écœurés de chaleur, de poussière et d'angoisse" (p. 733). Ainsi, s'il faut qualifier la macrostructure du Sursis de "classiquement linéaire", la microstructure mérite lappellation de "cinématographique", car elle est intrinsèquement parallèle et ce, grâce à l'esthétique du montage alterné, qui est révélateur de la simultanéité.

"Le monde est plein de signes. Tout est signe. Il faut savoir les déchiffrer» (p. 987). Cette conception sémiologique du monde, que nous devons à un personnage du Sursis, Philippe, est tout un programme qui renvoie au postulat d'Adorno: "Tout sens d'un texte littéraire est véhiculé par la forme " (Santerres-Sarkany, p. 59) ${ }^{26}$. Il s'agit donc ici d'une sémantique du discours.

Dans un roman comme Le Sursis, comme d'ailleurs dans la plupart des romans "collectifs", l'unité de la diégèse est assurée 
par le lecteur - unité étant à comprendre ici comme "cohérence». Il s'agit donc, pour le lecteur, de mettre à l'œuvre tout un dispositif mental qui sera le véritable ombilic et la membrane de la diégèse. Ce que l'esthétique de la réception allait penser découvrir à partir des années soixante, les formalistes russes l'ont reconnu très tôt, au plus tard dès les années vingt. Le concept de discours intérieur, que l'on doit à Boris Eikhenbaum, qualifie judicieusement le travail que le lecteur doit fournir pour mettre en relation le déluge de segments auquel il est confronté à la lecture du Sursis. Pour pouvoir raccorder sémantiquement entre eux les différents "morceaux" de l'histoire, produits des nombreux changements de séries événementielles, donc du changement permanent d'espace, le lecteur doit assurer la continuité du montage en prenant appui sur la mémoire discursive (intratextuelle). Devant ce rythme soutenu, il ne peut se reposer uniquement sur les mécanismes du texte écrit en prenant repère sur les variables, surtout les personnages, puisque le discours, comme nous avons pu le voir, fait que certains segments ne sont pas immédiatement distribuables. De cette apparente discontinuité naît, grâce à la participation du lecteur, la continuité. Jean-Pierre Esquenazi parle à juste titre du spectateur comme du "lieu de synthèse du film" (p. 43). Cette mise en évidence du travail du spectateur est tout aussi pertinente en ce qui concerne le lecteur: "Il nous semble que ce que le film produit comme sens naît des associations effectuées par le spectateur pendant la projection, et que le film, comme discours cohérent, isolé de sa réception, n'existe pas" (p. 12).

Puisque "[...] l'espace et le temps sont les repères familiers dans le cadre desquels nous interprétons ce que nous percevons du monde qui nous entoure" (Auffray, p. 7), le montage est le moyen idéal de l'homme pour transcender sa perception continue et linéaire du monde; il lui permet d'en révéler la conception telle qu'elle s'effectue en sa conscience ou en son imagination, ceci étant particulièrement vrai en ce qui concerne la simultanéité ubiquitaire dont il ne pourra empiriquement sans doute jamais faire l'expérience concrète. "Linear thinking is out. Parallel is in " (p. 165) ${ }^{27}$ peut-on lire dans Microserfs, le dernier roman du canadien Douglas Coupland, auteur du désormais 
mythique Generation $X$. Si cette "pensée parallèle " est propre à notre société de l'informa(tisa)tion, elle n'est pas neuve pour autant: Le Sursis, écrit à l'époque des balbutiements de l'informatique et des recherches sur la communication, ainsi que de lavènement des mass media, n'est-il pas déjà une vision littéraire de cette société de la communication en temps réel qui voit le jour à l'aube du troisième millénaire? Toute sa structure repose sur le parallélisme évoqué par Coupland, parallélisme qui, comme le rappelle Pierre Lévy - éminent spécialiste français du virtuel _- dans son article au titre très sartrien, "L'universel sans totalité », est la particularité de la cyberculture:

La cyberculture incarne la forme horizontale, simultanée, purement spatiale, de la transmission. Elle ne relie dans le temps que par surcroît. Sa principale opération est de se connecter dans l'espace, de construire et d'étendre les rhizomes du sens (p. 119).

Qu'il s'agisse de cyberculture, de cinéma ou de littérature, le rôle du récepteur / destinataire en tant quacteur sur la scène du sens est donc impératif. Sans ce cérébrisme, Le Sursis ne serait qu'un foisonnement d'instants disparates; par son travail de lecture cinématographique, le lecteur construit la simultanéité qui ne lui a été que suggérée par l'écriture cinématographique ${ }^{28} \mathrm{du}$ roman, puisque selon Sartre, «[...] l'imagination du spectateur n'a pas seulement une fonction régulatrice mais constitutive; elle ne joue pas, elle est appelée à recomposer l'objet beau pardelà les traces laissées par l'artiste» (1996, p. 54).

Concluons donc de manière sartrienne avec François Jost, pour qui " [...] la représentation des événements est moins dans les choses que dans sa [le spectateur] tête»(p. 83), et Sal Paradise, le narrateur autodiégétique du roman $O n$ the Road de Jack Kerouac, s'interrogeant: "What's heaven? what's earth?" et dont la seule réponse rejoint mon propos: "All in the mind" (p. 245).

Université d'Aix-la-Chapelle 


\section{NOTES}

1 Pour une analyse détaillée de l'américanisation de la France, voir Richard Kuisel, Seducing the French. The Dilemma of Americanization (Berkeley / Los Angeles / London: University of California Press, 1993) dans lequel l'auteur fait de nombreuses références à Sartre.

2 Sartre a d'ailleurs déclaré à ce propos: "I am not at all anti-American, neither do I understand what "anti-American" means" (New York Herald Tribune, 20 novembre 1946). Son soi-disant "antiaméricanisme" étant plutôt une réaction à une tendance mercantiliste et à l'influence de celle-ci sur les équilibres sociopolitiques qu'un rejec pur et simple et en bloc de la culture américaine.

3 Notons tout de mème que la rhétorique classique connaissait déjà la stychomythic, le dialogue sous forme de répliques vers par vers, son utilisation étant cependant limitée aux moments de grande tension de la tragédie.

4 Gérard Genette y fait brièvement allusion dans son Nouveau Discours du récit (Paris: Seuil, 1983, p. 49).

5 Il serait intéressant de comparer le "désir de guerre" dans Le Sursis et Le Voyage au bout de la nuit de Louis-Ferdinand Céline (1932). Voir à ce sujer Jacques Lecarme, "Sartre et Céline: deux violents dans le siècle", Magazine littéraire, n" 282 (1990), p. $41-44$

6 Il sagit ici de ce que l'on pourrait appeler une séquence d'introduction, qui a pour contenu l'attente des protagonistes principaux du roman.

7 Voir ci-dessous mon analyse de la même constellation, où la locution « au même instant" est cependant absente.

8 Il s'agit de la séquence qui suit celle de l'attente des protagonistes au début du roman, et que l'on pourrait intituler la «résolution des protagonistes face à l'Histoire».

9 Dans son excellent ouvrage sur le cinéma des premiers temps, La Lucarne de l'infini. Naissance du langage cinématographique (Paris: Nathan, 1991), Noël Burch analyse comment s'est institutionnalisée cette notion d'ubiquité. Voir également Charles Perraton et Nathalie Bouchard, “Montrer, dire et saisir l'espace dans le cinéma des premiers temps: le cas du Great Train Robbery", Cinémas, vol. 3, n"1 (1995), p. 9-27. 10) Je fais ici allusion à l'ouvrage de Rainer Winter, Der produktive Zuschauer. Medienaneignung als kultureller und ästhetischer Prozeß (München: Quintessenz, 1995).

II Le concept d'interface n'appartient plus uniquement à l'informatique, si bien que Francesco Casetti lui a réservé quelques pages dans son étude sur la réception filmique, D'un regard l'autre. Le film et son spectateur (Lyon: Presses Universitaires de lyon, 1990, p. 198-204). Un autre exemple: Hanjo Berressem a intitulé sa remarquable étude sur le romancier américain Thomas Pynchon, Pynchon's Poetics. Interfacing Theory and Text (Urbana / Chicago: University of Illinois Press, 1993).

12 D'autres romanciers ont déjà rompu avec le "psychologisme" caractéristique du roman français classique, par exemple Cocteau dans Thomas l'imposteur (1924) — à propos de l'" antipsychologisme" de ce roman, voir mon étude Cocteau et Radiguet. Etude comparée de leur création romanesque parallèle (Bonn: Romanistischer Verlag Jakob Hillcn, 1992). Brecht formulera plus tard cette conception du cinéma comme art phénoménologique: "Der Film (braucht) äußere Handlung und nichts introspektiv Psychologisches" (Gesammelte Werke, XVIII, 1967, Frankfurt a. M.: Suhrkamp, p. 170-171).

13 Le clip, le spot publicitaire, la vidéo et aujourd'hui, l'informatique (Internet, CD-ROM, etc.) ont également leur propre esthétique. Bien entendu, toutes ces esthétiques s'interpénètrent l'une l'autre, comme cela a été le cas pour les rapports cinématélévision dans les années cinquante et soixante. À ce propos, Yshaghpour évoque à raison le rôle décisif qu'a joué Jean-Luc Godard. 
14 I'expression "pivot scriptural" est de Jean-Luc Seylaz cité par Michel Contat dans sa "Notice" au Sursis (Sartre, Euvres romanesques, Paris, Gallimard, 1981, p. 1967). Voir aussi l'analyse que fait Contat de la séquence avec focalisation sur le nègre qui chante.

15 Cettc continuité est caratéristique de ce que l'on nomme communément "le cinćma classique hollywoodien». Il existe d'ailleurs des termes techniques qui soulignent cette continuité: continutity editing et continuity cutting. Pour une introduction au continutity editing, voir David Bordwell er Kristin Thompson, Film Art. An Introduction (New York: Mc Graw-Hill, 1979, p. 218-229).

16 Un exemple de syntagme: le texte allant de la première phrase du roman (p. 733) - "Seize heures trente à Berlin, quinze heures trente à Londres" - à "-Oui, dit Milan. Oui. Je suis raisonnable. Allez, monte!» (p. 739).

17 Même si les segments précédents ne sont pas explicitement situés dans le temps ils pourraient très bien, cux aussi, être simultanés avec le segment qui donne la nouvelle indication temporelle et donc être de nature prospective par rapport à celui-ci -, le lecteur les interprétera immanquablement comme antérieurs à ce nouveau "moment" puisque, en lisant un nouvel indice temporel, c'est seulement à ce niveau du texte quil prendra conscience de cette évolution. Spéculer le contraire me paraît futilc, car rien ne perrnettrait de déterminer avec certitude que les segments énoncés avant un indice temporel seraient simultanés par rapport à celui-ci. Cette thèse pourrait cependant être vérifiće dans une lecrure du Sursis axée sur cet aspect.

18 Un exemple de segment: "Le vieillard se leva, il traversa la pièce, les genoux raides, d'un pas noble et sautillant. Il dit: "Messieurs!" et il sourit affablement; il posa le document sur la table et en lissa les feuillets de son poing fermén (p. 734).

19 La simultanéité ne connaît que de très rares explicitations volontairement marquées dans Le Sursis. Les transitions du type "À quinze heures trente, Mathieu attendait encore, au bord d'un horrible avenir; au même instant, à seize heures trente, Milan n'avait plus d'avenir " (p. 734) sont donc plus l'exception que la règle.

20 "Leerstelle" signifie en allemand espace vide, blanc ou lacune. C'est Wolfgang lser qui en a fait un concept dans le cadre de la théoric sur la réception de la littérature (Der Akt des Lesens. Theorie ästhetischer Wirkung, München: Wilhelm Fink, 1976, p. 284 (t suiv.).

21 Contrairement à la littérature, le cinéma, la télévision et l'image en général semblent ètre considérés comme quelque chose que l'homme est capable de lire des son plus jeune âge. Cette faculté, Philippe Viallon la questionne dans son commentaire sur la télévision: "Mais où le téléspectateur a-t-il appris à "regarder" J'image en génćral et la télévision en particulier? Est-ce inné chez l'homme moderne? les comportements les plus élémentaires (marcher, shabiller, se nourrir) ont tous exigé un apprentissage. Les adultes ont-ils acquis cette compétence avec d'autres savoirs? Mais quand ct où? 'Tous les enfants ont-ils la chance d'avoir des parents, eux-mêmes compétents, qui leur expliquent le message télévisuel? Ou bien n’est-ce qu'un faux problème: tout le monde est bien capable de comprendre tellement le message télévisuel est simple! Pourtant la facilité de lecture de l'image, qu'elle soit fixe ou animée, est un lieu commun qui a fait long feu!" (L'Analyse du discours de la télévision, Paris: P.U.F. 1996, p. 5-6).

22 J'emprunte évidemment cette expression à l'ouvrage de John R. Searle, Les Actes de langage. Essai de philosophie du langage (Paris: Herman, 1972).

23 À noter que ce segment avec focalisation sur Jeannine est en continuité avec la scène de masturbation (p. 790-791), sur laquelle je reviendrai en détail plus loin.

24 Il serait cependant possible aussi au cinéma de "cacher" les informations pertinentes en nutulisant que des très gros plans. 
25 A plusieurs reprises, Sartre a recours à la focalisation zéro. Axant ainsi son discours sur un montage spatio-temporel parfois outrancier, Sartre renie en quelque sorte la liberté du personnage et favorise la focalisation zéro, annihilant ainsi d'une certaine manière le théorème existentialiste élégamment formulé par l'ćcrivain américain l'aul Auster: "Every man is the author of his own life" (Moon Palace. london / Boston: Faber\&Faber, 1990, p. 7).

26. Une lecture du Sursis axée uniquement sur cet aspect sémiologique et sur le postulat d'Adorno - que rejoint le fameux "the medium is the message" de Marshall McL uhan (Understanding Media. The Extensions of Man, Cambridge: The MIT Press, $1964, p .7)$ - pourrait révéler jusqu'à quel point il s'agit ici d'un chaos sémiologique où signifiant et signifié ne font plus qu'un.

27 Dans son roman précédent, on trouve déjà une représentation très intéressante, de type strictement parataxique, de la simultanéité: "The car radio's seck button was continuously prowling for new stations. Quirks in the Van Allen radiation belts allowed me to receive radio stations from all over the West - those fragments of culcural memory and information that compose the invisible information structure I consider my real home - my virtual community. I was hearing the sort of information that I knew would make me homesick if I were stuck in Europe or dying in Vietnam : it was $61^{\circ}$ in San Francisco and $58^{\circ}$ in Daly City; a Christian talk show from Las Vegas asked listeners to pray for a housewife with lupus; traffic on the Santa Monica Freeway was frozen owing to an overturned propane tanker at the Normandie onramp; the Mayor of Albuquerque was accepting listener phone-ins" (Life after Cod, New York: Pocket Books, 1994, p. 168).

28 Comme je le mentionnais plus haut, il faut garder à l'esprit que les compléments de temps renvoyant explicitement à la simultanéité (au même moment, etc.) sont largement absents du Sursis.

\section{OUVRAGES CITÉS}

Anders, Güinter. Die Antiquiertheit des Menchen, Band I. Über die Seele in Zcitalter der zweiten industriellen Revolution. München : Beck, 1988.

Auffray, Jean-Paul. L'Espace-temps. Paris: Flammarion, 1996.

Aumont, Jean-Jacques et Michel Marie. L'Analyse des films. Paris: Nathan, 1988.

Bessalel, Jean et André Gardies. 200 mots clés de la théorie du cinéma. Paris : Curf, 1992.

Bory, Jean-Louis. "Les Chemins de la liberté ". Arts et Lettres, n" 1 (1946), p. 81.

Brochier, Jean-Jacques. "Le contemporain capital". Magazine littéraire, "La passion des idées" (hors série), 1996, p. 72-74.

Brochier, Jean-Jacques. "Sartre le patron". Magazine littéraire, n" 225 (1985), p. 9-10. Chapman, Seymour. Story and Discourse. Narrative Structure in Fiction and Film. Ithaca : Cornell University Press, 1978.

Contat, Michel. "Notice sur Le Sursis", dans Jean-Paul Sartre, CEuvres romanesques. Paris: Gallimard (1981), p. 1963-1971.

Eisenstein, Serguei. "Montage 38 ", Le Film, sa forme, son sens. Paris: Bourgois (1976), p. 213-252.

Esquenazi, Jean-Pierre. Film, perception et mémoire. Paris: L'Harmattan, 1994.

Idt, Geneviève. "Les Chemins de la liberté. Les Tomboggans du romanesque". Obliques, n"1 18-19 (1979), p. 75-94.

Ishaghpour, Youssef. D'une image à l'autre. La nouvelle modernité du cinéma. La représentation du cinéma aujourd'hui. Paris: Denoël-Gonthier, 1982. 
Jost, François. "Direcr, narration, simultanéité: frontières de la temporalité». Cinćmas, vol. 5 , n"' 1-2 (1994), p. 81-90.

Kacmmerling, Ekkehard. «Die filmisch Schreibweise », dans Matthias Prangel (direction), Materialien zu Alfred Döblin "Berlin Alexanderplatz". Frankfurt am Main: Suhrkamp (1975), p. 185-198.

Koenot, Jan. Hungry for Heaven. Rockmusik, Kultur und Religion. Düsseldorf: Patmos, 1997.

L.crot, Jacques. Précis a'e linguistique générale. Paris: Minuit, 1993.

Lévy, Pierre. "L'universel sans totalité». Magazine littéraire, "La passion des idées" (hors séric), 1996, p. 116-119.

Metz, Christian. Essats sur la signification at cinéma, tome 1. Paris: Klincksieck, 1968.

Sartre, Jean-Paul. «Situation de l'écrivain en 1947 ", Situation II. Qu'est-ce que la littérature? Paris: Gallimard (1996), p. 169-295.

Sartre, Jean-Paul. "À propos de John Dos Passos er de 1919", Sitzation 1. Paris: Gallimard (1993), p. 14-24.

Sartre, Jean-Paul. "Le Sursis", Euvres romanesques. Paris: Gallimard, 1981.

Sartre, Jean-Paul. "L'universel singulier", Situation IX. Paris: Gallimard (1972), p. 152-190.

Sartre, Jean-Paul. "American Novelists in French Eyes". The Atlantic Monthly, vol. CLXXVIII, n" 2 (1946), p. 114-118. 